\title{
Environmental Chemicals in an Urban Population of Pregnant Women and their Newborns from San Francisco
}

\author{
Rachel Morello-Frosch ${ }^{1,2,{ }^{*} \text {, Lara J. Cushing }}{ }^{7}$, Bill M. Jesdale ${ }^{1}$, Jackie M. Schwartz ${ }^{3}$, \\ Weihong Guo ${ }^{4}$, Tan Guo ${ }^{4}$, Miaomiao Wang ${ }^{4}$, Suhash Harwani ${ }^{4}$, Syrago-Styliani E. \\ Petropoulou ${ }^{4}$, Wendy Duong ${ }^{4}$, June-Soo Park ${ }^{4}$, Myrto Petreas ${ }^{4}$, Ryszard Gajek ${ }^{5}$, Josephine \\ Alvaran $^{5}$, Jianwen She ${ }^{5}$, Dina Dobraca ${ }^{6}$, Rupali Das ${ }^{6}$, Tracey J. Woodruff ${ }^{3,}$ \\ ${ }^{1}$ Department of Environmental Science, Policy and Management, University of California, \\ Berkeley, California, USA \\ ${ }^{2}$ School of Public Health, University of California, Berkeley, California, USA \\ ${ }^{3}$ Program on Reproductive Health and the Environment, Department of Obstetrics, Gynecology \\ and Reproductive Sciences, University of California, San Francisco, California, USA \\ ${ }^{4}$ Environmental Chemistry Laboratory, Department of Toxic Substances Control, California \\ Environmental Protection Agency, Berkeley, California, USA \\ ${ }^{5}$ Environmental Health Laboratory, California Department of Public Health, Richmond, California, \\ USA \\ ${ }^{6}$ Environmental Health Investigations Branch, California Department of Public Health, Richmond, \\ California, USA \\ ${ }^{7}$ Department of Health Education, San Francisco State University, San Francisco, California, USA
}

\section{Abstract}

Exposures to environmental pollutants in utero may increase the risk of adverse health effects. We measured the concentrations of 59 potentially harmful chemicals in 77 maternal and 65 paired umbilical cord blood samples collected in San Francisco during 2010-11, including polychlorinated biphenyls (PCBs), organochlorine pesticides (OCPs), polybrominated diphenyl ethers (PBDEs), hydroxylated PBDEs (OH-PBDEs), and perfluorinated compounds (PFCs) in serum, and metals in whole blood. Consistent with previous studies, we found evidence that concentrations of mercury $(\mathrm{Hg})$ and lower-brominated PBDEs were often higher in umbilical cord blood or serum than in maternal samples (median cord:maternal ratio > 1), while for most PFCs and lead $(\mathrm{Pb})$, concentrations in cord blood or serum were generally equal to or lower than their maternal pair (median cord:maternal ratio $\leq 1$ ). In contrast to the conclusions of a recent review, we found evidence that several PCBs and OCPs were also often higher in cord than maternal serum (median cord:maternal ratio > 1) when concentrations are assessed on a lipid-adjusted basis. Our findings suggest that for many chemicals, fetuses may experience higher exposures than their mothers, and highlight the need to characterize potential health risks and inform policies aimed at reducing sources of exposure.

\footnotetext{
*Corresponding authors: rmf@ berkeley.edu or tracey.woodruff@ucsf.edu, Telephone: (510) 643-6358 or 415-476-3198.
} 


\section{Graphical Abstract}

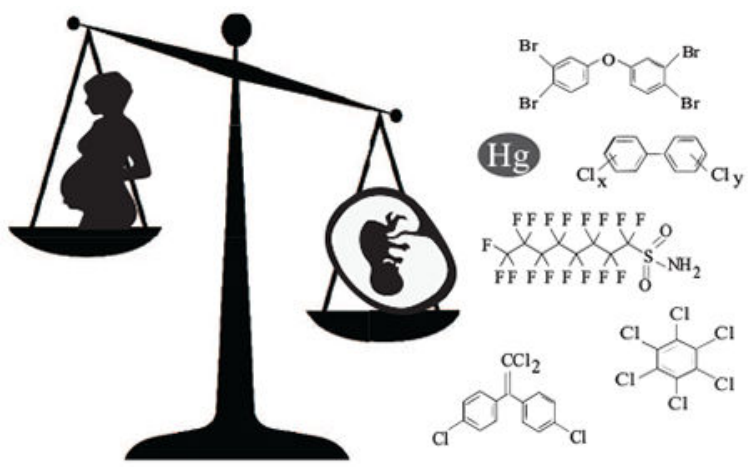

\section{Introduction}

Animal and human studies have linked prenatal exposure to environmental chemicals to adverse health effects both at birth (e.g. preterm birth, low birth weight, and birth defects) and later in life (e.g., neurodevelopmental defects, cancer, and cardiovascular disease). ${ }^{1,2}$ Previous research using National Health and Nutrition Examination Survey (NHANES) data found that pregnant women in the U.S. are exposed to numerous harmful manufactured chemicals, such as polychlorinated biphenyls (PCBs), organochlorine pesticides (OCPs), perfluorinated compounds (PFCs), industrial phenols, polybrominated diphenyl ethers (PBDEs), phthalates, and perchlorate. ${ }^{3}$ Many of these chemicals were detected in greater than $99 \%$ of U.S. pregnant women. ${ }^{3}$ Maternal exposures are of concern because many chemicals can cross the placenta to reach the fetal system ${ }^{4}$ and put the uniquely susceptible developing fetus ${ }^{5,6}$ at risk for adverse health outcomes. Health risks from simultaneous exposures to multiple chemicals are also of increasing concern, as co-exposures can have interactive adverse effects. ${ }^{7}$

Various factors can influence the extent to which chemicals enter the fetal environment, including chemical structure, protein-binding affinity, lipophilicity, and placental permeability. ${ }^{4}$ A recent review concluded that chemical concentrations in umbilical cord blood are generally lower than or equal to those in maternal blood, except in the cases of some brominated flame retardants, polycyclic aromatic hydrocarbons, magnesium, and mercury $(\mathrm{Hg})$, for which they are consistently higher in the fetus. ${ }^{8}$ However, estimates of maternal-to-fetal transfer efficiency varied widely across studies, often spanning an order of magnitude or more for the same chemical. The majority of studies reviewed also did not analyze maternal and fetal samples on a pair-wise basis, limiting the ability to assess interindividual variability in transfer efficiency.

The goal of this study was to better characterize prenatal exposures to multiple environmental chemicals among urban, primarily Latina women - a growing and important population that is not well represented in larger biomonitoring studies such as NHANES and to characterize individual variability in the transfer of chemicals between mother and fetus. We measured concentrations of a broad range of industrial chemicals and metals, including PFCs, PCBs, PBDEs and organochlorine pesticides, in paired maternal and 
umbilical cord blood samples collected from a convenience sample of pregnant women participating in the Chemicals in Our Bodies Study (CIOB Study, also referred to as the Maternal and Infant Environmental Exposure Project). Our research addresses limitations of previous studies of maternal-fetal transfer by analyzing maternal and cord samples on a pairwise basis and on a broader array of environmental chemicals.

\section{Materials and Methods}

\section{Study Population and Sample Collection}

The CIOB Study is a collaborative project of the California Environmental Contaminant Biomonitoring Program (or Biomonitoring California, www.biomonitoring.ca.gov) and the University of California (San Francisco and Berkeley) that measured chemical exposures in pregnant women seeking prenatal care at San Francisco General Hospital (SFGH) and their newborns. We enrolled 92 women from the SFGH Women's Health Center prenatal clinic during their second or third trimester of pregnancy between October 2010 and June 2011. At the time of enrollment into the CIOB Study, the Women's Health Center served predominantly low-income women of color (60\% Latina, 20\% African American, 12\% Caucasian and $8 \%$ Asian/Pacific Islander) who did not have private health insurance. Women were eligible to participate if they were English- or Spanish-speaking, 18 years or older, in their second or third trimester of pregnancy and if they did not have a high-risk pregnancy. CIOB Study protocols were approved by the Institutional Review Boards of the University of California, San Francisco (10-00861) and Berkeley (2010-05-04), and the California Health and Human Services Agency's Committee for the Protection of Human Subjects (10-04-05).

Demographic information was collected following recruitment and prior to delivery via interviewer-administered questionnaire. Maternal blood was collected during labor and delivery and umbilical cord blood after delivery and prior to umbilical cord clamping whenever possible. Blood was collected in ethylenediaminetetraacetic acid (EDTA)-coated tubes and stored at $-20^{\circ} \mathrm{C}$ until analyzed for metals. Blood was also collected in tubes without additives and, within 24 hours, serum was separated by allowing clotting at room temperature, then centrifuging twice at $2000 \mathrm{rpm}$ and transferring serum to amber glass vials for storage at $-20^{\circ} \mathrm{C}$ until analysis for persistent organic pollutants (POPs).

\section{Chemical Analysis}

We analyzed maternal and cord blood samples for 59 analytes: 15 PCBs, 7 OCPs, 19 PBDEs, 4 hydroxylated PBDEs (OH-PBDEs), and 11 PFCs in serum and 3 metals in whole blood (see Supporting Information for a full list of chemicals). Chemical analyses were conducted at the Biomonitoring California laboratories as described below. Method detection limits (MDLs) were defined as three times the standard deviation of the blank samples for persistent organic analytes in serum samples. For metal analysis, MDLs were defined as 3.14 times the standard deviation of archived blood specimens with known lowlevel of analytes. 


\section{PCBs, OCPs and PBDEs}

Our analytical method using gas chromatography/high resolution mass spectrometry (GCHRMS) was previously published ${ }^{9,10}$ and used in the current study with slight modifications. Thawed serum samples $(2 \mathrm{~mL})$ were spiked with carbon-labeled surrogate standards: nine ${ }^{13} \mathrm{C}$-labeled PCBs $\left({ }^{13} \mathrm{C}_{12}\right.$-PCB-101, $-105,118,-138,-153,-156,-170,-180$, and -194); seven ${ }^{13} \mathrm{C}$-labeled OCPs $\left({ }^{13} \mathrm{C}_{12}-2,4^{\prime}\right.$-DDT, ${ }^{13} \mathrm{C}_{12}-4,4^{\prime}$-DDE, ${ }^{13} \mathrm{C}_{12}-4,4^{\prime}$-DDT, ${ }^{13} \mathrm{C}_{6}$-hexachlorobenzene, ${ }^{13} \mathrm{C}_{10}$-oxychlordane, ${ }^{13} \mathrm{C}_{10}$-trans-nonachlor, and ${ }^{13} \mathrm{C}_{6}$ - $b$ hexachlorocyclohexane $[\mathrm{HCH}])$; and nine ${ }^{13} \mathrm{C}$-labeled PBDEs $\left({ }^{13} \mathrm{C}_{12}\right.$-BDE-28, -47, -99, $-153,-154,-183,-197,-207$, and -209$)$. Equal volumes (4 mL) of formic acid and water were added to each sample before loading on the solid phase extraction (SPE) modules (RapidTrace, Biotage ${ }^{\circledR}$, USA). Oasis HLB cartridges ( 3 cc, $500 \mathrm{mg}$, Waters, Inc. USA) and acidified silica $\left(500^{\circ} \mathrm{C}\right.$ pre-baked, manually packed, $\left.3 \mathrm{cc}\right)$ were used for the sample extraction and clean-up, respectively. The collected final eluates were concentrated and spiked with recovery standard $\left({ }^{13} \mathrm{C}_{12}\right.$-PCB-209). NIST standard reference material 1589a and bovine serum pre-spiked with known amounts of target analytes were used as quality assurance/quality control (QA/QC) samples. Blank samples (10 times diluted bovine serum) were also processed with each batch of samples.

We used GC-HRMS (DFS, ThermoFisher, Bremen, Germany) to measure PBDEs and PCBs/OCPs in two separate injections. For PCBs and OCPs analyses, we injected $2 \mu \mathrm{L}$ of extracts in splitless mode and separated them using a HT8-PCB column $(60 \mathrm{~m} \times 0.25 \mathrm{~mm}$ I.D., $0.25 \mu \mathrm{m}$ film thickness, SGE International Pty Ltd., Australia \& Pacific Region) with helium as carrier gas. For PBDEs analysis, we injected $2 \mu \mathrm{L}$ of extracts and separated them using a DB-5 MS column (Agilent J\&W, USA) $(15 \mathrm{~m} \mu 0.25 \mathrm{~mm}$ I.D., $0.10 \mu \mathrm{m}$ film thickness) with helium as carrier gas. The MS was operated in electron impact ionization mode using multiple ion detection. Perfluorokerosene (PFK) was used as the mass reference.

\section{Hydroxylated PBDEs (OH-PBDEs)}

An off-line SPE sample cleanup was implemented for the analysis of $250 \mu \mathrm{L}$ serum samples for OH-PBDEs, including a 3-hr enzymatic hydrolysis prior to extraction of the analytes. ${ }^{11}$ The SPE was performed using OASISTM HLB, $60 \mathrm{mg}, 3 \mathrm{cc}$ (Waters Inc., MA, USA) and the chromatographic separation was achieved on a mixed-mode column (Acclaim Surfactant Plus, $3 \mu \mathrm{m}, 2.1 \mathrm{~mm} \times 250 \mathrm{~mm}$; Thermo Scientific, Madison, WI, USA). An aliquot of $10 \mu \mathrm{L}$ of the reconstituted sample diluted four times was used for analysis. The analysis of $\mathrm{OH}-$ PBDEs in serum was carried out on a Prominence Ultra-Fast liquid chromatography system (UFLC) (Shimadzu Corporation, Columbia, MD, USA) interfaced with an AB Sciex 5500 Qtrap System (Applied Bioscience, Foster City, CA, USA) in triple quadrupole MS/MS mode.

Human serum pre-spiked with known amounts of target analytes were used as QC materials (low, medium and high) were processed with each batch of samples. Method and solvent blank samples were also processed with each batch and no OH-PBDEs were detected. 


\section{PFCs}

We used an online SPE high-performance liquid chromatography tandem MS (SPE-HPLCMS/MS) method. ${ }^{12}$ Briefly, $100 \mu \mathrm{L}$ of serum were mixed with $0.1 \mathrm{M}$ formic acid, and internal standards were added $\left({ }^{13} \mathrm{C}_{2}\right.$-perfluorooctanoic acid [PFOA] and ${ }^{13} \mathrm{C}_{4}$ perflucorooctane sulfonic acid [PFOS]), then injected by the online Symbiosis ${ }^{\mathrm{TM}}$ SPE-HPLC system (Symbiosis TM Pharma system with Mistral CS Cool, IChrom Inc.) to a C18 cartridge (HySphere C18 HD, $7 \mu \mathrm{m}, 10 \mathrm{~mm} \times 2 \mathrm{~mm}$ ). After washing, the target analytes were eluted to a C8 HPLC column (BETASIL C8 column, Thermo Fisher Scientific) for separation. The eluate was then introduced to the MS/MS (API 4000 QTrap, ABSciex) for multiple-reaction-monitoring (MRM) analysis. Analytes were quantified using a calibration curve constructed for each batch: regression coefficients of 0.98 to 0.99 were generally obtained.

In-house QC materials were prepared by spiking a known amount of PFC analytes in blank bovine serum at low and high levels. Standard reference materials (SRM 1958) from the National Institute of Standards and Technology (NIST, Gaithersburg, MD), and QC samples spiked with known PFC concentrations from the U.S. Centers for Disease Control and Prevention (CDC) were used as reference materials. Blank samples of bovine serum (Hyclone/GE Healthcare Life Sciences) were also processed with each batch of samples, and no PFCs were detected above their respective MDLs.

\section{Metals}

We analyzed whole blood specimens for total $\mathrm{Hg}$, cadmium $(\mathrm{Cd})$, and lead $(\mathrm{Pb})$, using an Agilent $7500 \mathrm{cx}$ inductively coupled plasma mass spectrometry system with a helium collision cell (Agilent Technologies, Inc., Folsom, CA). ${ }^{13}$ Blood specimens were diluted 1:50 prior to analysis with a diluent comprised of $4 \% \mathrm{w} / \mathrm{v}$ of n-butanol, $2 \% \mathrm{w} / \mathrm{v}$ of $\mathrm{NH} 4 \mathrm{OH}$, $0.1 \% \mathrm{w} / \mathrm{v}$ Triton X-100 and $0.1 \% \mathrm{w} / \mathrm{v}$ of H4EDTA to minimize blood matrix effects. Intermediate calibration standards were prepared from stock standard solutions traceable to the NIST.

Specimen concentrations were determined using calibration curves established during each analytical run, with regression coefficients $\geq 0.998$ for each analyte. Each specimen was analyzed in duplicate and the final result was calculated by averaging the two. Acceptance criteria were based on the relative percent difference (RPD) between the two specimens. The average result was deemed acceptable if the RPD was $\_2 \%$. Fewer than $1 \%$ of the reported samples had RPDs $>20 \%$ due to issues with sample clotting, especially with cord blood specimens. RPDs for these exceptions were $<35 \%$, and the average RPDs for $\mathrm{Cd}, \mathrm{Pb}$ and $\mathrm{Hg}$ were $11.3 \%, 3.7 \%$ and $6.4 \%$, respectively. RPDs were not considered when analytical values were below the MDL. Values were only reported for specimens with concentrations above the MDLs, and for specimens with analyte values below MDL levels, these were reported as $<$ MDL.

QC reference materials were prepared by spiking defibrinated sheep blood obtained from Hemostat Laboratories (Dixon, CA, USA) with stock standard solutions at three concentrations (low, medium and high). All reference materials were analyzed at both the 
beginning and end of each batch analysis. Four concentrations of NIST standard reference material 955c were periodically analyzed throughout the study to assure independent confirmation. In addition, method blanks were checked daily for any detectable levels of the analytes of interest.

\section{Lipids}

Cholesterol and triglycerides were enzymatically determined at Boston Children's Hospital (Boston, MA) and the total lipid content calculated. ${ }^{14}$

\section{Statistical Analysis}

We examined distribution plots and calculated summary statistics (detection frequency, geometric mean and 95th percentile) for concentrations of each chemical in both maternal and umbilical cord blood samples. We also calculated the conditional probability of detection in umbilical cord samples, given the detection of the chemical in the maternal sample. Some chemical concentrations were below the MDL in maternal and/or umbilical cord samples, resulting in left-censored data. Therefore, we used nonparametric methods to examine the correlation and transfer efficiency between maternal-umbilical cord pairs. We used rank-based Spearman's correlation coefficient to measure the association between paired maternal and umbilical cord concentrations, incorporating censored observations by assigning them tied ranks. We present conditional probabilities of detection and correlation coefficients for chemicals that were detected in at least 20 paired maternal samples in the main text; for the remaining chemicals this information is included in the Supporting Information.

We characterized transfer efficiency by calculating umbilical cord:maternal ratios of chemical concentrations among paired samples, conditional on the chemical being detected in the maternal sample. We estimated summary statistics of these ratios (percentiles, geometric mean $[\mathrm{GM}]$ and geometric coefficient of variation [GCV]) using nonparametric Kaplan-Meier survival analysis methods. ${ }^{15-17}$ The distribution of cord:maternal ratios that results when the MDL/ $\sqrt{2}$ is substituted for observations $<$ MDL are also provided in the Supporting Information for comparison. Statistical analysis were conducted using SAS 9.4 (SAS Institute Inc., Cary, NC) and the NADA package in R 3.2.2. ${ }^{18}$

\section{Results}

Our population consisted primarily of Latina women, and $95 \%$ of participants for whom we had income data had a combined household income of less than $\$ 40,000$ (see Supporting Information). Two-thirds were foreign-born (about one-third in Mexico). Maternal blood samples were successfully collected from 77 women (84\% of enrolled) and umbilical cord blood samples were successfully collected after 65 of these women ( $71 \%$ of enrolled) delivered their babies. Due to inadequate sample volume, only 55 umbilical cord samples were analyzed for the full panel of 59 chemicals.

We detected a median of 25 chemicals in maternal blood samples (range $15-40$ or $2568 \%$ of chemicals measured), and a median of 17 chemicals (range $11-27$ or 19-46\% of chemicals measured) in the 55 umbilical cord blood samples that were tested for the full panel of 59 
chemicals (Figure 1). Eight (14\%) of the 59 chemicals analyzed were detected in $\geq 90 \%$ of both maternal and umbilical cord samples: the OCPs $4,4^{\prime}$-dichlorodiphenyldichloroethene (4,4' -DDE) and hexachlorobenzene (HCB); the PFCs perfluorononanoic acid (PFNA), PFOS, PFOSA, and 2-(N-methyl-perfluorooctane sulfonamido) acetic acid (N-MeFOSAA); and the heavy metals $\mathrm{Pb}$ and $\mathrm{Hg}$ (see Supporting Information, which also includes summary statistics). Ten chemicals were detected in maternal but not umbilical cord samples; two chemicals (the PBDE 2,2',4' -tribromodiphenyl ether [BDE-17], and the BDE metabolite $6^{\prime}$ hydroxy-2,2' $, 4,4^{\prime}, 5$-pentabromodiphenyl ether [6'-OH-BDE-99]) were detected exclusively in a small number of umbilical cord samples (see Supporting Information).

We found that 30 chemicals were detected in at least 20 out of 65 paired maternal samples (Table 1). Among these 30 chemicals, the probability of a chemical's detection in umbilical cord serum or blood, given detection in its maternal pair, ranged from 8 to $73 \%$ for PCBs, 7 to $100 \%$ for OCPs, 0 to $68 \%$ for PBDEs, 46 to $88 \%$ for OH-PBDEs, 81 to $100 \%$ for PFCs, and 0 to $100 \%$ for metals (Table 1). Eighteen (60\%) and nine (30\%) of these 30 chemicals had conditional probabilities $\geq 50 \%$ and $\geq 90 \%$, respectively (Table 1). Conditional probabilities of detection in umbilical cord blood samples were higher for hydrophilic (median $=90 \%)$ than lipophilic (median=40\%) chemicals (Wilcoxon rank-sum pvalue $=0.004)$.

We found that lipid-adjusted and wet-weight chemical concentrations in umbilical cord samples were positively correlated at $\mathrm{p}<0.05$ with those in maternal samples for $23(77 \%)$ of the 30 chemicals detected in at least 20 paired maternal samples (Table 1). Statistically significant correlation coefficients ranged from 0.40 to 0.93 . The mean correlation coefficient was highest for metals (excluding Cd), then PFCs > OCPs > OH-PBDEs >

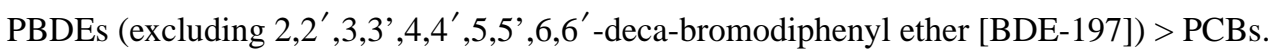
Twenty one $(70 \%)$ and six $(20 \%)$ of these 30 chemicals had correlation coefficients $>0.5$ and $>0.8$, respectively. Correlations between chemical concentrations in maternal and umbilical cord samples were higher for hydrophilic (median $\rho=0.79$ ) than lipophilic (median $\rho=0.53$ on a lipid-adjusted basis, $\rho=0.56$ on a wet-weight basis) chemicals (Wilcoxon rank sum $\mathrm{p}$-value $=0.04$ ). We also observed statistically significant correlation between maternal and cord concentrations of several chemicals that were not detected in at least 20 paired maternal samples, including PCBs, PBDEs, $2,4^{\prime}$ - and $4,4^{\prime}$-dichlorodiphenyltrichloroethane (DDT), and perfluorodecanoic acid (PFDeA), with coefficients ranging between 0.26 and 0.72 (see Supporting Information Table S4).

We found that ratios between chemical concentrations in paired maternal and cord samples varied by chemical class (Table 2, Figure 2). For lipophilic compounds, ratios varied by whether they were calculated on a lipid-adjusted or wet-weight basis (Figure 2A). On a wetweight basis, we found that median ratios of lipophilic compounds were typically lower than one, with median ratios ranging between 0.2 and 0.5 for PCBs; 0.1 and 0.6 for OCPs; and 0.2 and 0.4 for PBDEs (Table 2). Median ratios on a lipid-adjusted basis were between 0.7 and 1.4 for PCBs; 0.9 and 1.8 for OCPs; and 0.5 and 1.3 for PBDEs (Table 2). Whether using wet weight or lipid-adjusted concentrations, median ratios of PCB and PBDE concentrations decreased with the degree of halogenation, albeit not always linearly. For example, the median lipid-adjusted ratios of PCB-118 (5 chlorines), -138 and -153 (6 
chlorines), -170 and -180 ( 7 chlorines) were $1.4,1.4,1.1,0.7$, and 0.7 , respectively. The median lipid-adjusted ratios of BDE-28 ( 3 bromines), -47 (4 bromines), -99 and -100 (5 bromines), -153 (6 bromines), and -209 (10 bromines) were 1.2, 1.3, 1.0, 0.9, and 0.5, respectively (Table 2 ).

Most median PFC cord:maternal concentrations ratios were near or below one (Figure 2 and Table 2). For carboxylate PFCs, median ratios decreased with increasing chain length and degree of halogenation. The median ratio was $1.0,0.8,0.4$, and 0.3 for perfluoroheptanoic acid (PFHpA [C7, 13 fluorines]), PFOA (C8, 15 fluorines), PFNA (C9, 17 fluorines) and PFUA (C11, 21 fluorines), respectively (Table 2).

Individual-level variation in transfer efficiency, assessed using the GCV, tended to increase somewhat with an increase in the degree of halogenation for PCBs, OCPs, and carboxylate PFCs (Table 2). The median GCVs was lower for hydrophilic chemicals (median GCV=58) than lipophilic chemicals (median $\mathrm{GCV}=74$ on a lipid-adjusted basis, $\mathrm{GCV}=71$ on a wetweight basis) but the differences were not statistically significant at $\mathrm{p}<0.10$.

Substituting observations $<$ MDL with MDL $/ \sqrt{2}$ generally resulted in slightly higher median cord:maternal ratios and GCVs on both a wet-weight and lipid-adjusted basis (see Supporting Information).

\section{Discussion}

To our knowledge, this is the first study to measure nearly 60 environmental chemicals in matched maternal and umbilical cord blood samples in the U.S. We found widespread exposures to a mixture of different chemicals in this primarily Latina and largely lowincome population. All but $12(21 \%)$ of the 56 chemicals detected in maternal blood samples were also detected in umbilical cord blood samples, indicating that they passed through the placenta and entered the fetal environment, and we observed statistically significant and moderate-to-strong correlation between maternal and umbilical cord concentrations for the majority (77\%) of chemicals detected in at least 20 paired maternal samples. Further, we found that concentrations of four chemicals (the PBDE metabolite 5-OH-BDE-47, the PFCs PFOSA and 2-(N-ethylperfluorooctane sulfonamido) acetic acid [N-EtFOSAA], and $\mathrm{Hg}$ ), were more often higher in umbilical cord serum or blood than in maternal samples from the same woman (i.e., the median cord:maternal concentration ratios were greater than one). Median cord:maternal concentration ratios also exceeded one for many lipophilic compounds (PCB-118, -138 and -153, 4, $4^{\prime}$-DDE, HCB, and BDE-28 and -47) when ratios were calculated on a lipid-adjusted basis.

Chemical concentrations in maternal blood samples from our study population were generally lower than those measured in a study of pregnant women in the U.S. derived from NHANES $^{3}$ although our sample included more extreme observations (higher $95^{\text {th }}$ percentiles) for 4,4'-DDE, BDE-47 and BDE-99, and PFNA (See Supporting Information Table S3). Lower average concentrations among CIOB Study participants are likely due to the differences in study periods: the NHANES data were collected in 2003-2004, while our samples were collected between 2010 and 2011. Levels of some bioaccumulative 
compounds are steadily decreasing in the U.S. population following regulatory bans and voluntary phase-outs. ${ }^{19,20}$ For example, bans and phase-outs of certain PBDEs have led to declines in concentrations measured in pregnant women. ${ }^{9}$ Differences in measured concentrations may also reflect differences in geographic origin of the study populations. Two-thirds of the women in our study were foreign-born (primarily from Mexico or Central America), and previous studies indicate that immigrants have lower PBDE concentrations compared to U.S.-born women in California. ${ }^{21,22}$ This may be attributable to the state's unique furniture flammability standard, which has likely contributed to higher PBDE concentrations among women who have been in California longer. The use of DDT and other organochlorine pesticides was also banned earlier in the U.S. compared to other countries in the Americas, which may explain the higher $95^{\text {th }}$ percentile $4,4^{\prime}$-DDE concentrations observed in our study compared with pregnant women in NHANES 2003-4.

In general, we found much higher estimates of maternal-fetal transfer (cord:maternal ratios) for lipophilic compounds (PCBs, OCPs, and PBDEs) when ratios were calculated on a lipidadjusted rather than a wet-weight basis. This is due to the fact that the umbilical cord blood had lower concentrations of lipids than maternal blood, consistent with previous studies. ${ }^{23,24}$ If one assumes body burden equilibrium in which lipid-adjusted serum measurements correspond to concentrations in adipose tissue of the mother and fetus, comparisons on a lipid-adjusted basis are appropriate. However, if certain chemical exposures are themselves associated with higher lipid concentrations in blood, ${ }^{25}$ then this may complicate the underlying relationship between maternal-fetal transfer of chemicals and lipid adjustment may be less appropriate. ${ }^{26}$ Moreover, differences in lipid concentrations between maternal and cord blood preclude comparisons of transfer efficiency across contaminants, which would need to be done on a molar basis.

Differences in chemical elimination half-lives and placental transfer efficiency due to the chemical structures of the compounds are likely to have influenced the variation in maternalfetal ratios we observed across chemicals. Wet weight cord:maternal concentration ratios of PCBs in our study slightly exceeded those in previous studies that have analyzed paired maternal and umbilical cord samples. ${ }^{23,27,28}$ We found that cord:maternal concentration ratios decreased with increasing degree of halogenation of PCBs, suggesting that greater halogenation may result in lower fetal exposures. At least one other study has found evidence of a similar trend with chlorination of $\mathrm{PCBs},{ }^{27}$ while others have found little evidence of such a trend. ${ }^{23}$

Kim et al. (2015) observed a greater accumulation of PBDEs in umbilical cord serum as compared to PCBs and other polychlorinated organic compounds and hypothesized that a unique transplacental transfer mechanism related to the structural similarity of PBDEs to thyroid hormone may account for this difference. ${ }^{29}$ However, we did not find evidence that transplacental transfer of PBDEs was markedly higher than those of PCBs or OCPs. Our findings are consistent with the majority of previous studies finding lower transfer of lower brominated PBDEs relative to higher brominated PBDEs, ${ }^{30-33}$ although this also has not been found in all studies. ${ }^{29,34}$ Our estimates of transfer efficiency of the OCPs $4,4^{\prime}$-DDE, $\mathrm{HCB}$, and b-HCH are similar to those of a previous study conducted in Mexico. ${ }^{35}$ 
Although we measured generally lower concentrations of PFCs than previous studies of maternal and cord serum, ${ }^{24,36,37}$ our cord:maternal concentration ratios for PFOA and PFNA were consistent with those studies. Our finding that cord:maternal concentration ratios decreased with the increasing chain length of the perfluoroalkyl carboxylates is also consistent with one previous study of PFOA (C-8), PFNA (C-9) and perfluorodecanoic acid (PFDA [C-10]). ${ }^{36}$ We observed higher cord:maternal ratios for PFOS than were found in previous studies. ${ }^{24,36,37}$ Transfer efficiency appears to be higher for more branched isomers of both PFOA and PFOS, ${ }^{36}$ but we did not differentiate isomers in our study so we were unable to ascertain whether differences in branching may explain the higher transfer efficiency we observed for PFOS. We found that PFOSA and N-Et-FOSAA had slightly higher transfer efficiencies than other PFCs, which to our knowledge has not been documented before.

Cadmium was detected in $83 \%$ of maternal samples in this study, but not in any cord blood samples, although MDLs were the same. This is consistent with prior research indicating that the placenta serves as a partial barrier for $\mathrm{Cd}$, perhaps through metallothiopein binding, 38,39 although some studies have detected $\mathrm{Cd}$ in cord blood. ${ }^{8}$ Strong correlations between cord and maternal concentrations of $\mathrm{Hg}$ and $\mathrm{Pb}$ were observed in this study, similar to past work, ${ }^{8}$ and provides further evidence that concentrations in maternal blood taken at delivery are a good measure of relative fetal exposures to $\mathrm{Pb}$ and $\mathrm{Hg}$ at delivery.

Chemical concentrations were determined using blood samples taken at delivery. It is possible that maternal:cord correlations and ratios vary throughout pregnancy due to changes in body mass index (BMI), plasma volume expansion (PVE), lipid transfer, bone mobilization, and behavioral factors. Previous research found an inverse relationship between weight gain during pregnancy and concentrations of POPs in pregnant women. ${ }^{40}$ Other studies indicate that plasma volume during pregnancy progressively increases until 30-34 weeks gestation, when it reaches a plateau. This process may dilute chemical concentrations of metals in blood, ${ }^{41}$ although there has been no systematic review of the evidence of such dilution. There is also a redistribution of lipids from mother to fetus during the last trimester of pregnancy, ${ }^{34,42}$ which could result in greater fetal exposure to lipophilic compounds during the latter part of pregnancy. We were not able to assess the impact of BMI, PVE or lipid transfer on our maternal and neonatal exposure estimates due to a lack of data on these measures.

We used nonparametric methods to obtain unbiased estimates of correlation and central tendency in the presence of missing values below the MDL. This results in estimates of transfer efficiency that are conservative because they are conditional on detection in the maternal sample and in some cases omit cord/maternal pairs where chemicals were detected in cord samples but not in maternal samples (see Supporting Information Table S5 for the higher estimates that result from the substitution of missing values with the MDL $/ \sqrt{ } 2$, which allows for the inclusion of these pairs). Alternate approaches to analyzing left-censored data include discarding missing values, the substitution of missing values with a fixed value, maximum likelihood estimate (MLE), and multiple imputation. Omitting censored observations discards valuable information (i.e. that missing observations are known to be below the MDL) and leads to biased estimates of central tendency ${ }^{43}$ Substitution with a 
fixed value is problematic because the unobserved values are likely to be of various concentrations below the MDL. MLE and multiple imputation are sensitive to assumptions about the underlying distribution of the data. They are generally not recommended when working with less than 50 uncensored observations because of the difficulty of assessing whether the assumed distribution is reasonable. ${ }^{43}$

Our analysis did not examine the potential health consequences of chemical exposures. While human studies have examined health effects for individual congeners or groups of congeners of the compounds we studied, to our knowledge, no human studies have examined the potentially adverse developmental and reproductive health effects of simultaneous exposures to multiple chemicals in utero, which can have greater risks compared to individual exposures, particularly for the same adverse health endpoint. ${ }^{44}$ Exposures to many of the chemicals we measured are known to affect similar endpoints, such as maternal thyroid hormone disruption (e.g., PCBs and PBDEs) ${ }^{45}$ and adverse neurodevelopmental outcomes (PCBs, PBDEs, Pb and Hg). ${ }^{46}$ This study, combined with other evidence of ubiquitous exposures to multiple environmental chemicals during a sensitive period of development, highlights the need to better characterize the potential health risks of prenatal exposures, which would inform policies aimed at reducing sources of exposures.

\section{Supplementary Material}

Refer to Web version on PubMed Central for supplementary material.

\section{Acknowledgments}

Funding was provided by the California Wellness Foundation, the Centers for Disease Control and Prevention (Cooperative Agreement 5U38EH000481), the U.S. Environmental Protection Agency (EPA) Science to Achieve Results Fellowship (91744701-01), grant RD83543301 from the U.S. EPA, and P01 ES022841 from the National Institute of Environmental Health Sciences. Biomonitoring California is implemented by the California Department of Public Health, the Office of Environmental Health Hazard Assessment, and the Department of Toxic Substances Control. In addition to the named authors, we acknowledge the contributions of the Biomonitoring California program staff.

\section{References}

(1). Stillerman KP; Mattison DR; Giudice LC; Woodruff TJ Environmental exposures and adverse pregnancy outcomes: a review of the science. Reprod Sci 2008, 15 (7), 631-650; DOI 10.1177/1933719108322436. [PubMed: 18836129]

(2). Gluckman PD; Hanson MA Living with the past: evolution, development, and patterns of disease. Science 2004, 305 (5691), 1733-1736; DOI 10.1126/science.1095292. [PubMed: 15375258]

(3). Woodruff TJ; Zota AR; Schwartz JM Environmental chemicals in pregnant women in the United States: NHANES 2003-2004. Environ. Health Perspect 2011, 119 (6), 878-885; DOI 10.1289/ ehp.1002727. [PubMed: 21233055]

(4). Barr DB; Bishop A; Needham LL Concentrations of xenobiotic chemicals in the maternal-fetal unit. Reprod. Toxicol 2007, 23 (3), 260-266; DOI 10.1016/j.reprotox.2007.03.003. [PubMed: 17386996]

(5). Casals-Casas C; Desvergne B Endocrine disruptors: from endocrine to metabolic disruption. Annu. Rev. Physiol 2011, 73, 135-162; DOI 10.1146/annurev-physiol-012110-142200. [PubMed: 21054169] 
(6). Schug TT; Janesick A; Blumberg B; Heindel JJ Endocrine disrupting chemicals and disease susceptibility. The Journal of Steroid Biochemistry and Molecular Biology 2011, 127 (3-5), 204215; DOI 10.1016/jjsbmb.2011.08.007. [PubMed: 21899826]

(7). Kortenkamp A Ten years of mixing cocktails: a review of combination effects of endocrinedisrupting chemicals. Environ. Health Perspect 2007, 115 (S1), 98-105; DOI 10.1289/ehp.9357. [PubMed: 18174957]

(8). Aylward LL; Hays SM; Kirman CR; Marchitti SA; Kenneke JF; English C; Mattison DR; Becker RA Relationships of chemical concentrations in maternal and cord blood: a review of available data. J Toxicol Environ Health B Crit Rev 2014, 17 (3), 175-203; DOI 10.1080/10937404.2014.884956. [PubMed: 24749481]

(9). Zota AR; Linderholm L; Park J-S; Petreas M; Guo T; Privalsky ML; Zoeller RT; Woodruff TJ Temporal Comparison of PBDEs, OH-PBDEs, PCBs, and OH-PCBs in the Serum of Second Trimester Pregnant Women Recruited from San Francisco General Hospital, California. Environmental Science \& Technology 2013, 47 (20), 11776-11784; DOI 10.1021/es402204y. [PubMed: 24066858]

(10). Guo W; Nelson D; Hurley S; Reynolds P; Guo T; Wang M; Park J-S; Petreas M Pilot Study to Assess Effects of Collection Tube Types and Processing Delay on Measurements of Persistent Organic Pollutants and Lipids in Human Serum. Chemosphere 2014, 116, 75-82; DOI 10.1016/ j.chemosphere.2014.04.007. [PubMed: 24792582]

(11). Petropoulou S-SE; Duong W; Petreas M; Park J-S Fast liquid chromatographictandem mass spectrometric method using mixed-mode phase chromatography and solid phase extraction for the determination of 12 mono-hydroxylated brominated diphenyl ethers in human serum. J Chromatogr A 2014, 1356, 138-147; DOI 10.1016/j.chroma.2014.06.048. [PubMed: 25001336]

(12). Wang M; Park J-S; Petreas M Temporal changes in the levels of perfluorinated compounds in California women's serum over the past 50 years. Environ. Sci. Technol 2011, 45 (17), 7510 7516; DOI 10.1021/es2012275. [PubMed: 21732675]

(13). Gajek R; Barley F; She J Determination of essential and toxic metals in blood by ICP-MS with calibration in synthetic matrix. Analytical Methods 2013, 5 (9), 2193; DOI 10.1039/c3ay26036d.

(14). Phillips DL; Pirkle JL; Burse VW; Jr JTB; Henderson LO; Needham LL Chlorinated hydrocarbon levels in human serum: Effects of fasting and feeding. Arch. Environ. Contam. Toxicol 1989, 18 (4), 495-500; DOI 10.1007/BF01055015. [PubMed: 2505694]

(15). Helsel DR Statistics for Censored Environmental Data Using Minitab® and R; John Wiley \& Sons, Inc.: Hoboken, NJ, USA, 2011.

(16). Kirkwood T Geometric means and measures of dispersion. Biometrics 1979, 35 (4), 908-909.

(17). Bland MJ; Altman DG Measurement error proportional to the mean. British Medical Journal 1996, 313 (7049), 106; DOI 10.1136/bmj.313.7049.106. [PubMed: 8688716]

(18). R Core Team. R: A Language and Environment for Statistical Computing; R Foundation for Statistical Computing: Vienna, Austria, 2013.

(19). Ma W-L; Yun S; Bell EM; Druschel CM; Caggana M; Aldous KM; Buck Louis GM; Kannan K Temporal Trends of Polybrominated Diphenyl Ethers (PBDEs) in the Blood of Newborns from New York State during 1997 through 2011: Analysis of Dried Blood Spots from the Newborn Screening Program. Environ. Sci. Technol 2013, 47 (14), 8015-8021; DOI 10.1021/es401857v. [PubMed: 23755886]

(20). Nost TH; Breivik K; Fuskevag O-M; Nieboer E; Odland JO; Sandanger TM Persistent Organic Pollutants in Norwegian Men from 1979 to 2007: Intraindividual Changes, Age-Period-Cohort Effects, and Model Predictions. Environmental Health Perspectives 2013, 121 (11-12), 12921298; DOI 10.1289/ehp.1206317. [PubMed: 24007675]

(21). Zota AR; Rudel RA; Morello-Frosch RA; Brody JG Elevated house dust and serum concentrations of PBDEs in California: unintended consequences of furniture flammability standards? Environ. Sci. Technol 2008, 42 (21), 8158-8164; DOI 10.1021/es801792z. [PubMed: 19031918]

(22). Eskenazi B; Chevrier J; Rauch S; Kogut K; Harley KG; Johnson C; Trujillo C; Sjodin A; Bradman A In Utero and Childhood Polybrominated Diphenyl Ether (PBDE) Exposures and 
Neurodevelopment in the CHAMACOS Study. Environmental Health Perspectives 2012, 121 (2), 257-262; DOI 10.1289/ehp.1205597. [PubMed: 23154064]

(23). Park J-S; Bergman A; Linderholm L; Athanasiadou M; Kocan A; Petrik J; Drobna B; Trnovec T; Charles MJ; Hertz-Picciotto I Placental Transfer of Polychlorinated Biphenyls, Their Hydroxylated Metabolites and Pentachlorophenol in Pregnant Women from Eastern Slovakia. Chemosphere 2008, 70 (9), 1676-1684; DOI 10.1016/j.chemosphere.2007.07.049. [PubMed: 17764717]

(24). Needham LL; Grandjean P; Heinzow B; J0rgensen PJ; Nielsen F; Patterson DG; Sjodin A; Turner WE; Weihe P Partition of Environmental Chemicals between Maternal and Fetal Blood and Tissues. Environ. Sci. Technol 2011, 45 (3), 1121-1126; DOI 10.1021/es1019614. [PubMed: 21166449]

(25). Lee D-H; Steffes MW; Sjodin A; Jones RS; Needham LL; Jacobs DR Jr. Low Dose Organochlorine Pesticides and Polychlorinated Biphenyls Predict Obesity, Dyslipidemia, and Insulin Resistance among People Free of Diabetes. PLoS ONE 2011, 6 (1), e15977; DOI 10.1371/journal.pone.0015977. [PubMed: 21298090]

(26). Schisterman EF; Whitcomb BW; Buck Louis GM; Louis TA Lipid Adjustment in the Analysis of Environmental Contaminants and Human Health Risks. Environ Health Perspect 2005, 113 (7), 853-857; DOI 10.1289/ehp.7640. [PubMed: 16002372]

(27). Covaci A; Jorens P; Jacquemyn Y; Schepens P Distribution of PCBs and organochlorine pesticides in umbilical cord and maternal serum. The Science of the Total Environment 2002, 298, 45-53; DOI 10.1016/S0048-9697(02)00167-5. [PubMed: 12449328]

(28). Tsukimori K; Morokuma S; Hori T; Takahashi K; Hirata T; Otera Y; Fukushima K; Kawamoto T; Wake N Characterization of placental transfer of polychlorinated dibenzo-p-dioxins, dibenzofurans and polychlorinated biphenyls in normal pregnancy. Journal of Obstetrics and Gynaecology Research 2013, 39 (1), 83-90; DOI 10.1111/j.1447-0756.2012.01906.x. [PubMed: 22672617]

(29). Kim J-T; Son M-H; Lee D-H; Seong WJ; Han S; Chang Y-S Partitioning Behavior of Heavy Metals and Persistent Organic Pollutants among Feto-Maternal Bloods and Tissues. Environmental Science \& Technology 2015, 49 (12), 7411-7422; DOI 10.1021/es202408a. [PubMed: 26000703]

(30). Frederiksen M; Thomsen C; Frashaug M; Vorkamp K; Thomsen M; Becher G; Knudsen LE Polybrominated diphenyl ethers in paired samples of maternal and umbilical cord blood plasma and associations with house dust in a Danish cohort. International Journal of Hygiene and Environmental Health 2010, 213 (4), 233-242; DOI 10.1016/j.ijheh.2010.04.008. [PubMed: 20471317]

(31). Guvenius DM; Aronsson A; Ekman-Ordeberg G; Bergman A; Noren K Human prenatal and postnatal exposure to polybrominated diphenyl ethers, polychlorinated biphenyls, polychlorobiphenylols, and pentachlorophenol. Environ Health Perspect 2003, 111 (9), 12351241. [PubMed: 12842779]

(32). Chen A; Park J-S; Linderholm L; Rhee A; Petreas M; DeFranco EA; Dietrich KN; Ho S Hydroxylated Polybrominated Diphenyl Ethers in Paired Maternal and Cord Sera. Environ. Sci. Technol 2013, 47 (8), 3902-3908; DOI 10.1021/es3046839. [PubMed: 23506475]

(33). Meijer L; Weiss J; van Velzen M; Brouwer A; Bergman A; Sauer PJJ Serum Concentrations of Neutral and Phenolic Organohalogens in Pregnant Women and Some of Their Infants in The Netherlands. Environ. Sci. Technol 2008, 42 (9), 3428-3433; DOI 10.1021/es702446p. [PubMed: 18522129]

(34). Foster WG; Gregorovich S; Morrison KM; Atkinson SA; Kubwabo C; Stewart B; Teo K Human maternal and umbilical cord blood concentrations of polybrominated diphenyl ethers. Chemosphere 2011, 84 (10), 1301-1309; DOI 10.1016/j.chemosphere.2011.05.028. [PubMed: 21663933]

(35). Waliszewski SM; Aguirre AA; Infanzon RM; Siliceo J Partitioning Coefficients of Organochlorine Pesticides Between Mother Blood Serum and Umbilical Blood Serum. Bulletin of Environmental Contamination and Toxicology 2000, 65 (3), 293-299; DOI 10.1007/ s001280000127. [PubMed: 10903351] 
(36). Beesoon S; Webster GM; Shoeib M; Hamer T; Benskin JP; Martin JW Isomer Profiles of Perfluorochemicals in Matched Maternal, Cord, and House Dust Samples: Manufacturing Sources and Transplacental Transfer. Environmental Health Perspectives 2011, 119 (11), 16591664; DOI 10.1289/ehp.1003265. [PubMed: 21757419]

(37). Gutzkow KB; Haug LS; Thomsen C; Sabaredzovic A; Becher G; Brunborg G Placental transfer of perfluorinated compounds is selective - A Norwegian Mother and Child sub-cohort study. International Journal of Hygiene and Environmental Health 2012, 215 (2), 216-219; DOI 10.1016/j.ijheh.2011.08.011. [PubMed: 21937271]

(38). Baranowska I Lead and cadmium in human placentas and maternal and neonatal blood (in a heavily polluted area) measured by graphite furnace atomic absorption spectrometry. Occup Environ Med 1995, 52 (4), 229-232; DOI 10.1136/oem.52.4.229. [PubMed: 7795737]

(39). Sakamoto M; Yasutake A; Domingo JL; Chan HM; Kubota M; Murata K Relationships between trace element concentrations in chorionic tissue of placenta and umbilical cord tissue: Potential use as indicators for prenatal exposure. Environ Int 2013, 60C, 106-111; DOI 10.1016/j.envint. 2013.08.007.

(40). Bradman ASA; Schwartz JM; Fenster L; Barr DB; Holland NT; Eskenazi B Factors predicting organochlorine pesticide levels in pregnant Latina women living in a United States agricultural area. J Expo Sci Environ Epidemiol 2007, 17 (4), 388-399; DOI 10.1038/sjjes.7500525. [PubMed: 17033681]

(41). Faupel-Badger JM; Hsieh C-C; Troisi R; Lagiou P; Potischman N Plasma volume expansion in pregnancy: implications for biomarkers in population studies. Cancer Epidemiol. Biomarkers Prev 2007, 16 (9), 1720-1723; DOI 10.1158/1055-9965.EPI-07-0311. [PubMed: 17855687]

(42). Pipe NG; Smith T; Halliday D; Edmonds CJ; Williams C; Coltart TM Changes in fat, fat-free mass and body water in human normal pregnancy. Br J Obstet Gynaecol 1979, 86 (12), 929-940. [PubMed: 118770]

(43). Helsel DR More Than Obvious: Better Methods for Interpreting Nondetect Data. Environmental Science \& Technology 2005, 39 (20), 419A-423A.

(44). Phthalates and Cumulative Risk Assessment: The Task Ahead; National Academies Press: Washington, D.C., 2008; http://www.nap.edu/catalog/12528.

(45). Crofton KM Thyroid disrupting chemicals: mechanisms and mixtures. Int. J. Androl 2008, 31 (2), 209-223; DOI 10.im/j.1365-2605.2007.00857.x. [PubMed: 18217984]

(46). Science and Decisions: Advancing Risk Assessment, National Academies Press: Washington, D.C., 2009; http://www.nap.edu/catalog/12209. 


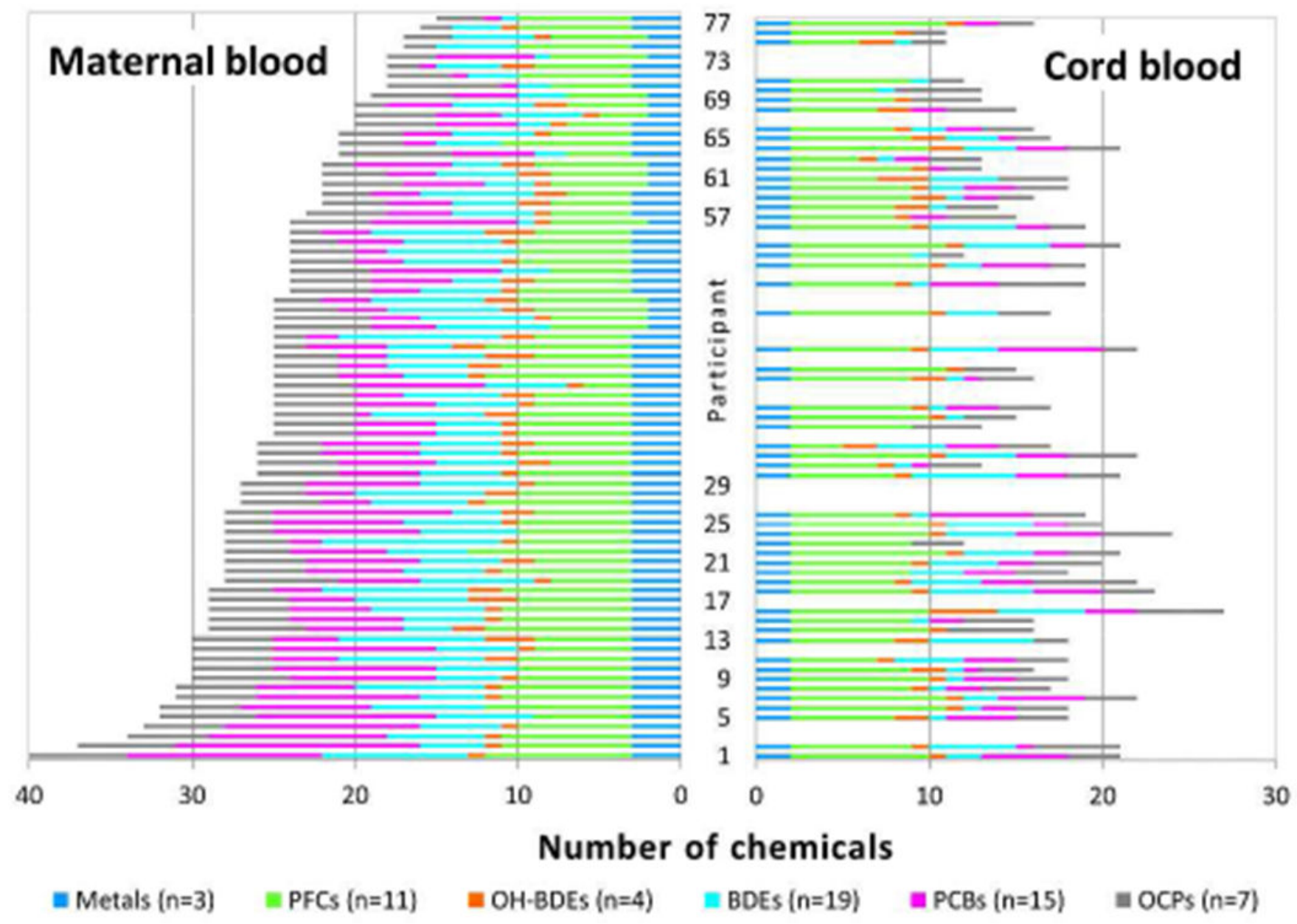

Figure 1.

Frequency plot of the number of chemicals detected in 77 maternal and 55 matched umbilical cord blood samples from the Chemicals in Our Bodies Study. ${ }^{a}$

a 65 umbilical cord samples were collected. Only the 55 samples that were tested for the full panel of 59 chemicals are shown. 

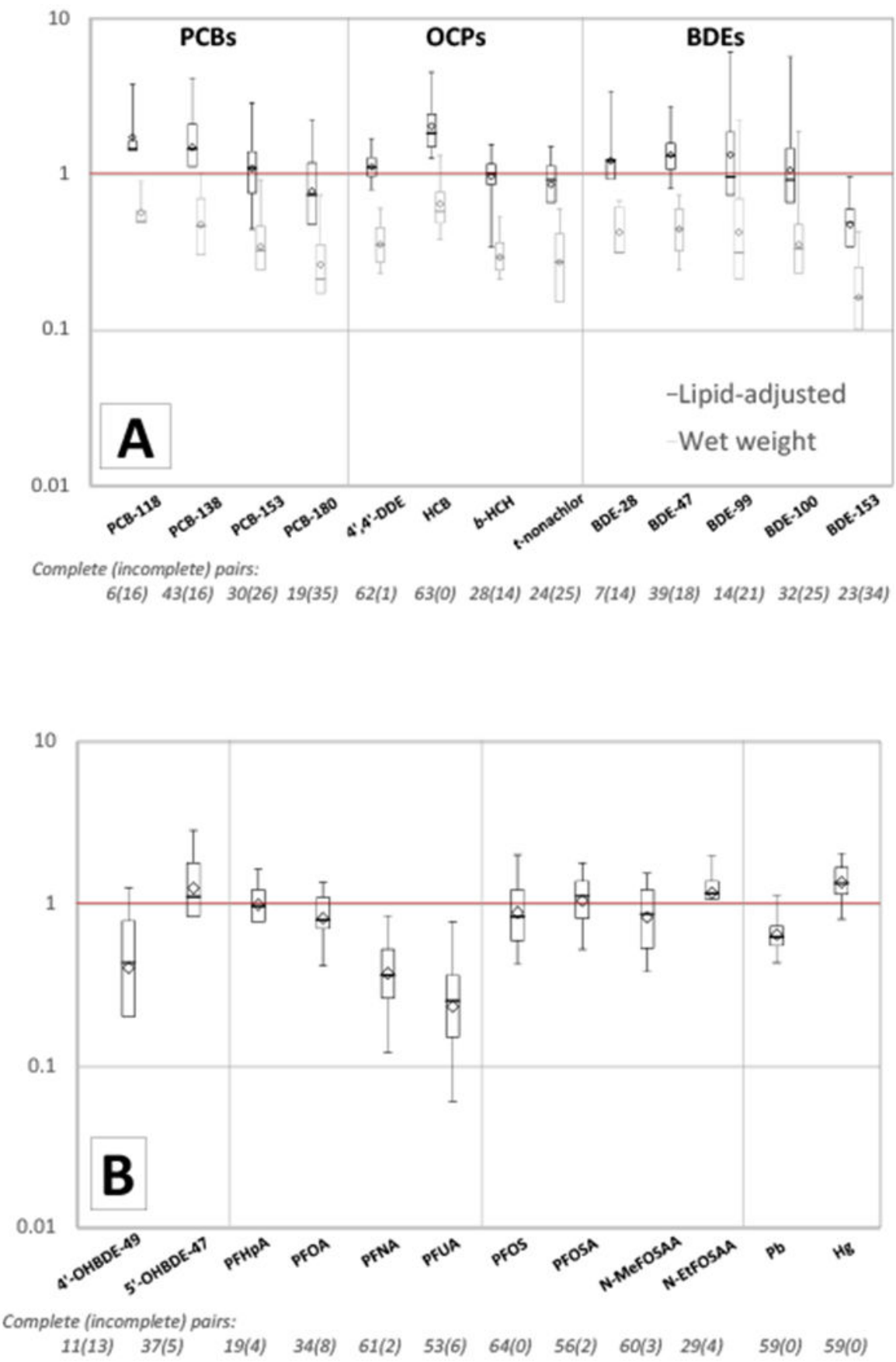

Figure 2.

Umbilical cord:maternal concentration ratios of A) lipophilic compounds (PCBs, OCPs, and PBDEs), and B) hydrophilic compounds (OH-PBDEs, PFCs and metals) detected in at least 20 of 65 paired maternal samples of whole blood (metals) or serum (all others). ${ }^{\mathrm{a}}$

Ratios were estimated using nonparametric survival analysis methods and are conditional on detection in the maternal sample. The bold horizontal line indicates the median ratio and the diamond indicates the geometric mean. The box delineates the interquartile range $\left(25^{\text {th }}\right.$ to $75^{\text {th }}$ percentile); whiskers extend to the $5^{\text {th }}$ and $95^{\text {th }}$ percentiles when they could be 
calculated. The number of incomplete cases refers to the number of paired observations where the measured concentration was $\geq \mathrm{MDL}$ in the maternal sample but $<\mathrm{MDL}$ in the cord sample.

a BDE-197 and Cd are omitted because they were not detected in any cord samples.

PCB-170, oxychlordane, and BDE-209 are omitted because they were detected in too few cord samples to calculate a $25^{\text {th }}$ percentile. 


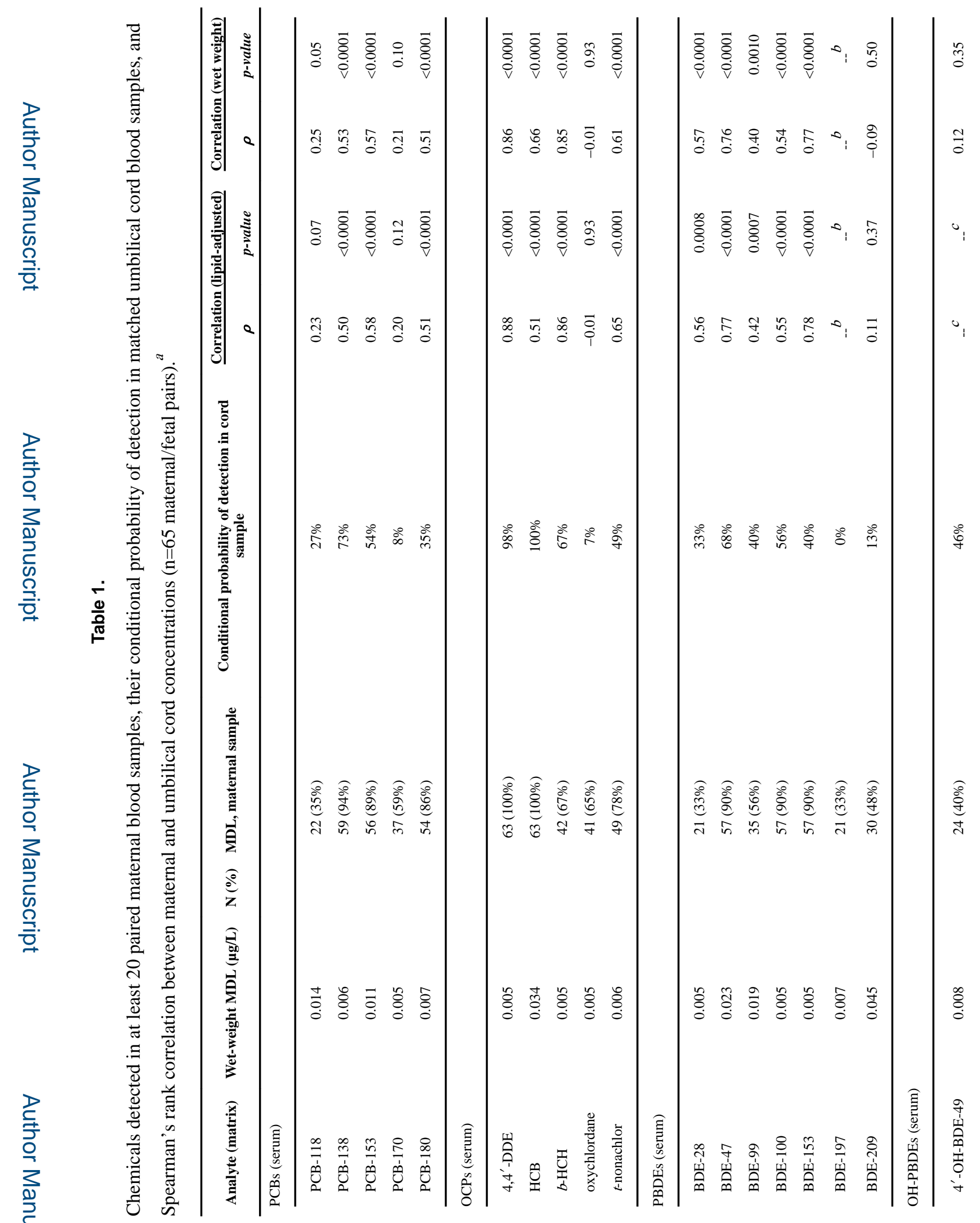


Morello-Frosch et al.

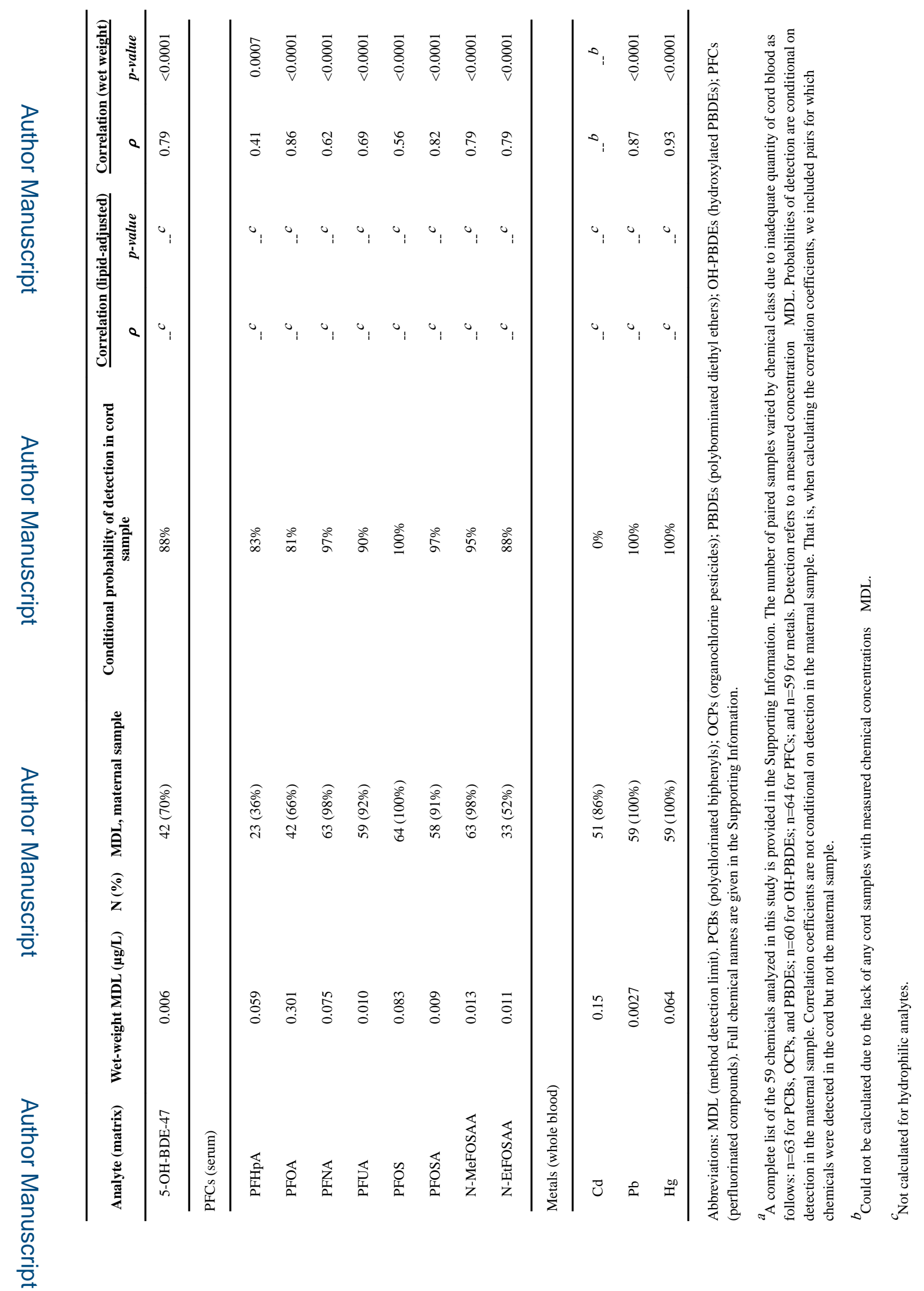

Environ Sci Technol. Author manuscript; available in PMC 2019 August 05. 


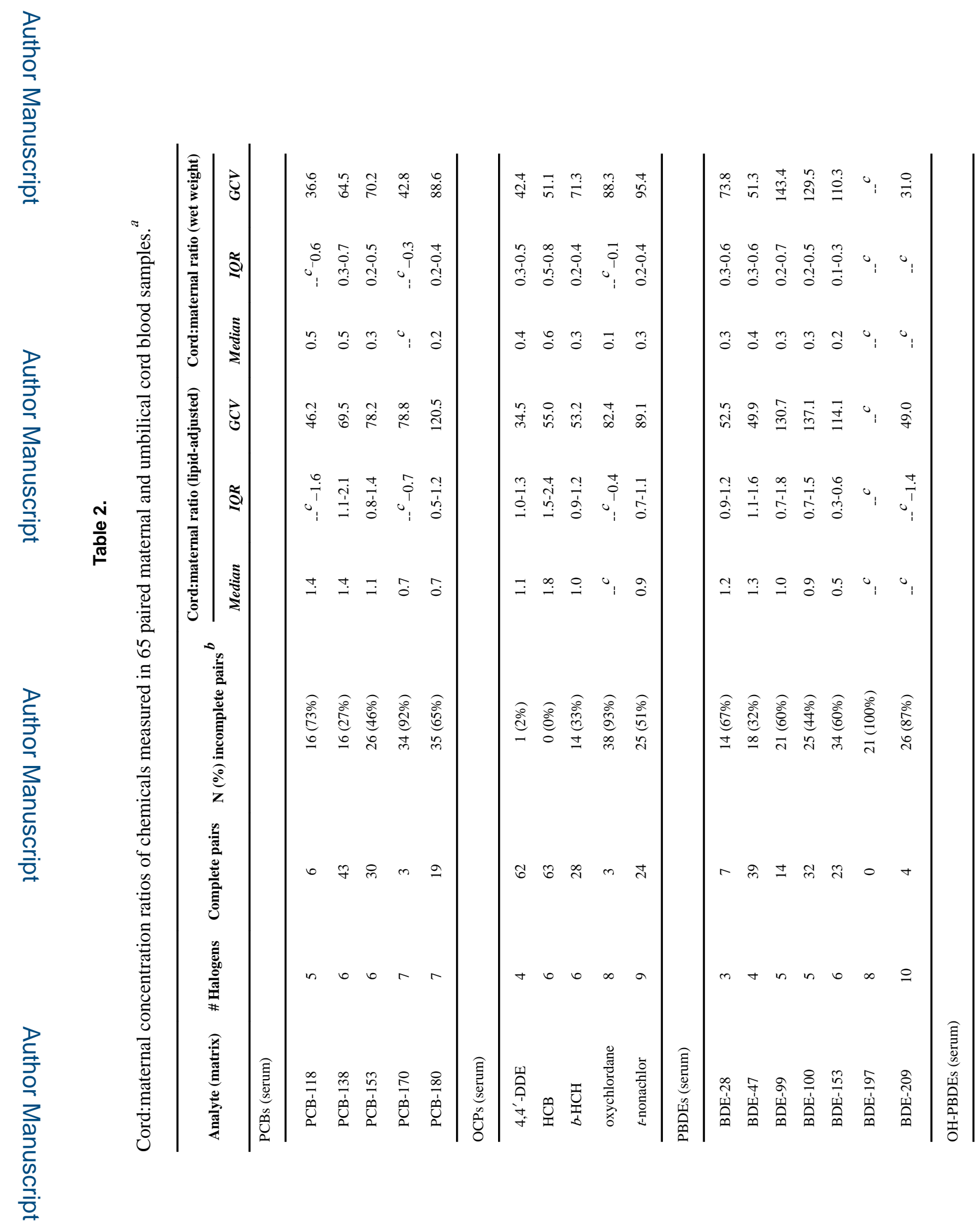

Environ Sci Technol. Author manuscript; available in PMC 2019 August 05. 


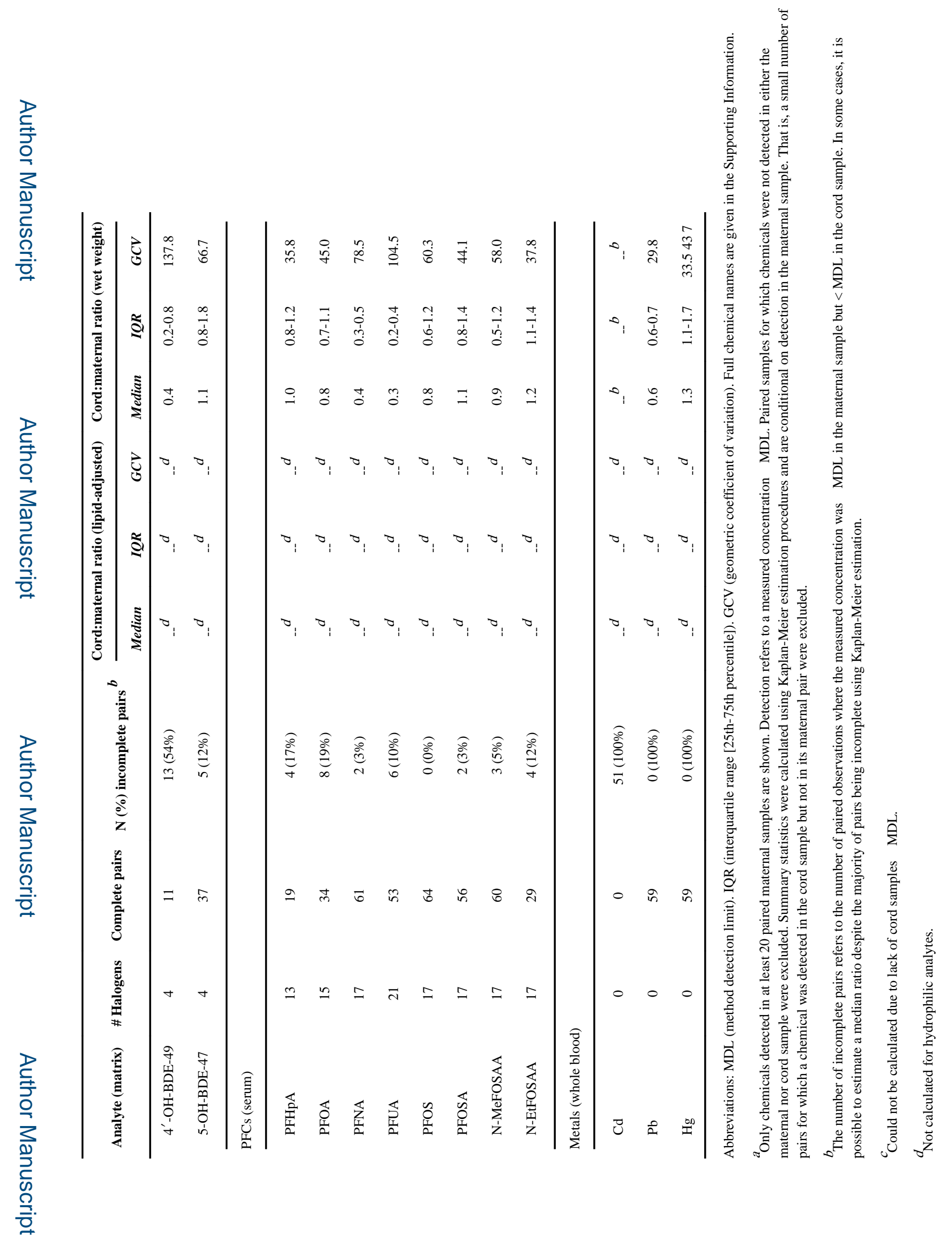

Environ Sci Technol. Author manuscript; available in PMC 2019 August 05. 